\title{
Image Fusion Techniques: A Review
}

\author{
Dhirendra Mishra, PhD \\ Associate Professor, \\ Department of Computer Engineering \\ MPSTME, SVKM's NMIMS University, Mumbai, \\ Maharashtra 400056, India
}

\author{
Bhakti Palkar \\ Ph.D. Scholar, MPSTME, \\ SVKM'S NMIMS University, \\ Mumbai, 400056, Maharashtra, India. \\ Associate Professor. KJSCE, \\ Mumbai, 400077, Maharashtra, India
}

\begin{abstract}
Image Fusion is used to retrieve important data from a set of input images and put it into a single output image to make it more informative and useful than any of the input images. It improves quality and applicability of data. Quality of the fused image depends on the application. Image fusion is widely used in intelligent robots, stereo camera fusion, medical imaging, and manufacture process monitoring, electronic circuit design and inspection, complex machine/device diagnostics and in intelligent robots on assembly lines. This paper presents a literature review on various spatial and frequency domain image fusion techniques such as averaging, min-max, block replace, HIS,PCA, brovey, pyramid based and transform based techniques. Various quality measures have been discussed to perform quantitative comparison of these methods.
\end{abstract}

\section{General Terms}

Digital Image Processing

\section{Keywords}

Image Fusion, Spatial Domain, Frequency Domain Techniques, Wavelets etc.

\section{INTRODUCTION}

Image Fusion reads several images of the same scene or objects and retrieves important information from them to put it into a single output image. Image generated by this method always gives more information than any of the input images which thereby improves quality and applicability of data. The definition of "quality" depends on the particular application area. In recent years there has been tremendous improvement in the image acquisition techniques. Latest technologies in image capturing devices help us to extract variety of different information from an image. This information can be collectively combined using "fusion" to generate more informative image. Multi-view, multi-modal, multi-temporal and multi-focus are the four ways in which image fusion can be performed. Mono modal images captured at the same time but from various viewpoints can be fused multi-view fusion methods. Multi-modal fusion is performed on images captured using various sensors. Multi-temporal fusion is performed on images of the same scene but captured at different times. Multi-focus fusion is performed on images captured with various focal lengths. Image fusion has been used in many applications. Image fusion is widely used in remote sensing. In satellite images are of two types: Panchromatic images and Multispectral images. Using fusion these two images can be merged to produce a single high resolution multispectral image. In medical applications, Image fusion is commonly used term. Medical images from the same modality or multiple modalities such as CT, MRI, SPECT, PET can be merged using fusion to generate a more informative image which can help in diagnosis and treatment. Image fusion is used in intelligent robots to gain motion control, based on feedback from various sensors. Manufacturing process can use fusion technique in inspection, measurement, monitoring and diagnostics of components or processes. Image fusion research work started with spatial domain image fusion techniques such as averaging, minimum, maximum, block averaging and principal component analysis (PCA) etc. Then it was soon succeeded by pyramid based fusion and discrete transform based fusion. Pyramid based fusion techniques are based on filters whereas discrete transform based fusion methods are based on transforms like DCT, wavelet etc. Figure 1 shows various types of image fusion techniques reviewed in this paper.

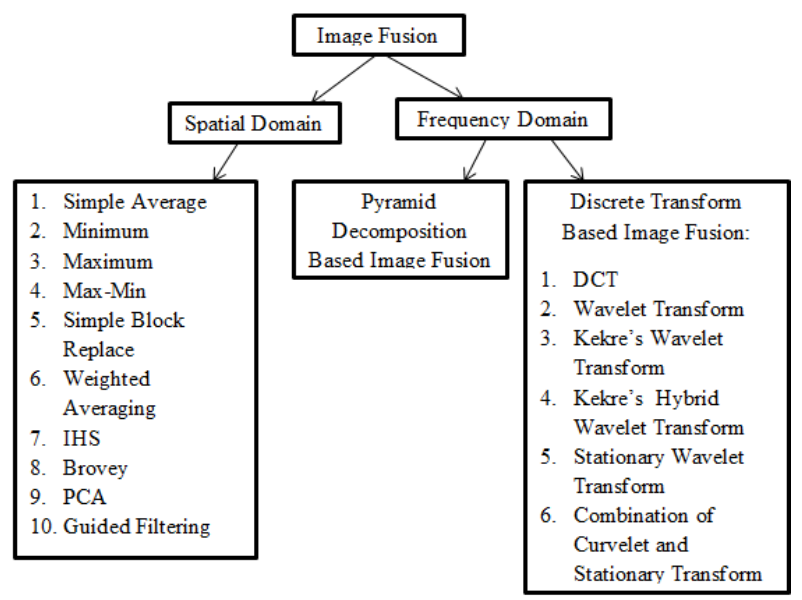

Fig 1: Image Fusion Techniques

This paper is organized as follows: Section two focuses on Spatial Domain Techniques. Section three is for Pyramid Based Fusion. Section five is for Discrete Transform Based Fusion. Section five gives performance measure parameters of Fusion techniques. Conclusion is presented in Section six.

\section{SPATIAL DOMAIN TECHNIQUES}

All Spatial Domain Techniques are also called as simple image fusion techniques.

Jasiunaset al. [5] and Dong et al. [6] used Simple Average, Select Maximum Pixel Value, Select Minimum Pixel Value, Select Max-Min and Simple Block Replace Techniques to fuse images. Out of these methods Simple average is basic fusion method in which the fused image is obtained by averaging corresponding pixels in the input images. Select maximum/minimum pixel value techniques select maximum/minimum pixel from corresponding pixels in the input images to generate fused image. In max-min technique 
fused image is obtained by averaging smallest and largest values of corresponding pixels in all input images. Simple block replace technique is based on pixel neighbourhood. In every image for each pixel (x,y), its neighbouring pixels are added and a block average is calculated. Pixel $(x, y)$ of the fused image is obtained by taking pixel with maximum block average among all corresponding pixels in input images. All these methods are very simple to understand and easy to implement. Simple average technique reduces the resultant image quality consequently by introducing noise into fused image. It leads to unwanted side effects like reduced contrast. Other methods in this category produce blurred output which in turn affects the contrast of the image. Therefore these techniques are not suitable for real time applications.

Song et. Al [7] suggested weighted averaging technique in which different weights are assigned to all source images. Fused image Pixel $(\mathrm{x}, \mathrm{y})$ is obtained by computing weighted sum of all corresponding pixels in input images. It improves detection reliability but increases signal to noise ratio of the fused image.

Principal Component Analysis (PCA) [9][10] is a statistical procedure that converts a set of observations of possibly correlated variables into a set of values of uncorrelated variables called principal components. The number of principal components is less than or equal to the number of original variables. Naidu et al. [8] and sadjadi [11] have used PCA for Image Fusion. It is very easy to use for image fusion and fused image has high spatial quality. But it also results in spatial degradation.

IHS is a color space, where 'hue' is the predominant wavelength of a color, 'saturation' is total amount of white light of a color and 'intensity' relates to the total amount of light that reaches the eye. Because of its simplicity and high sharpening ability, many applications using IHS transform in image fusion have been reported. Harris et al. [4] described how to use IHS in integrating Radar with diverse types of data such as Landsat TM, airborne geophysical and thematic data. However, there exists color distortion in the fused image because IHS assumes that the intensity is formed by even contribution from the RGB bands; thus, all the details contained in the high resolution image are directly integrated into the intensity component.

Gillespie et al. [3] have shown that Brovey Transform should be used to produce high contrast RGB image. Like HIS, Brovey works with only three bands.

Shutao [2] has presented an image fusion method which uses the average filter to get the two-scale representations decomposition of an image into a base layer containing large scale variations in intensity, and a detail layer capturing small scale details. It is very simple, computationally efficient. It can well preserve the original and complementary information of multiple input images, making it quite qualified for real applications.

\section{PYRAMID BASED FUSION}

Sadjadi et al. [11] and sanju kumara et al. [12] have explained Image Fusion Techniques Based on Pyramid Decomposition. A pyramid based fusion comprises of a number of images at different scales which together represent the original image. Every pyramid based image fusion has three stages. Number of levels ' $\mathrm{L}$ ' of pyramid is pre decided based on the size of the image.
In stage one which is repeated ' $\mathrm{L}$ ' times, original image is convolved with a predefined filter of the corresponding method and a pyramid is formed for that level. Input images are then decimated to half their sizes. In second stage, using the final decimated input matrices a new image matric is generated either by selecting minimum or maximum or taking average. Third stage is repeated ' $L$ ' times wherein input image matrix is un-decimated and then convolved with the transpose of the filter used in stage one. Filtered matrix is merged with the pyramid formed at the respective level of stage one by the process of pixel intensity value addition. The resultant image goes as input to the next level. The merged image of the last level is always the resultant fused image.

Burt et al.[14] and Olkkonen et al. [15] have explained Gaussian Pyramid and Laplacian Pyramid. Burt[16] has explained gradient pyramid. Toet [17] has explained ratio of low pass pyramid .Anderson [18] has explained filtersubtract-decimate hierarchical pyramid. Ramac [19] has explained Morphological filters pyramid with the help of opening and closing operations. Image closing enhances the sharpness of the edges of the objects in the images but at the same time it degrades the overall quality of the image. Ratio Pyramid based fusion is inferior to Morphological pyramid method. The ratio between the corresponding pixels of the input images and the filtered image does not really help in enhancement of the sharpness of an image. Other pyramid decomposition based fusion methods produce more or less similar output. Pyramid Methods provide good visual quality of an image for multi-focus images. The number of decomposition levels affects image fusion result.

\section{DISCRETE TRANSFORM BASED FUSION [20]}

Algorithm for Transform Based Fusion Techniques is as follows:

Step 1: Separate their RGB planes if the input images are coloured, to perform 2D transforms.

Step 2: Apply various transform techniques like KWT, DCT, Hybrid Walsh-DCT, etc.

Step 3: Compute average of corresponding pixels to obtain fused transform components.

Step 4: Apply inverse transform to convert fused transform components into image.

Step 5: Combine separated RGB planes if the input images were colored.

The diagram for discrete transform based fusion scheme is shown in Fig.1.

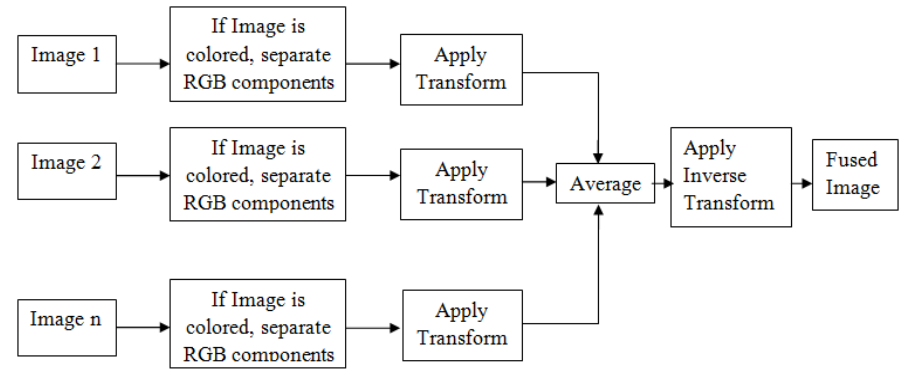

Fig 2: Discrete Transform Based Fusion 


\subsection{Discrete Cosine Transform (DCT) based Image Fusion}

Naidu et al. [17] have come up with six different types of image fusion techniques using DCT namely - DCTav, DCTma, DCTah, DCTch, DCTe and DCTcm. Steps for Image fusion using 'DCT' are as follows:

1. Divide input images into non-overlapping blocks of size $\mathrm{N} \times \mathrm{N}$.

2. Repeat following steps for every block.

a. Compute DCT coefficients for each block

b. Apply fusion rules to get fused DCT coefficients.

c. Apply IDCT on the fused coefficients.

In DCTav, DCTma, DCTe and DCTcm methods, DC components are averaged together. In DCTah and DCTch methods, the lowest AC components including DC coefficients are averaged. In DCTma and DCTah methods AC coefficients are chosen based on largest magnitude. In DCTch and DCTcm method AC coefficients are chosen based on largest contrast measure where as in DCTe method AC coefficients are chosen based on largest energy frequency band.

These techniques do not perform well while using the algorithms with block size less than $8 \times 8$ and also the block size equivalent to the image size itself. DCTav is very simple and basic image fusion technique in DCT domain. DCTe and DCTmx based image fusion algorithms performed well. These algorithms are very simple and can be used for real time applications.

\subsection{Discrete Wavelet Transform Method (DWT)}

The Discrete Wavelet Transform Method (DWT)[22] of an image $\mathrm{x}$ is calculated by passing it through a series of filters. First it is passed through a low pass filter impulse response $\mathrm{g}$ and is also decomposed simultaneously using a high-pass filter $h$. The outputs give the detail coefficients (from the high-pass filter) and approximation coefficients (from the low-pass).This decomposition is repeated to further increase the frequency resolution and the approximation coefficients decomposed with high and low pass filters and then down sampled. Images are fused using the fusion rules. Inverse Discrete Wavelet Transform is applied on fused decomposed level to generate fused image. The 'Haar' wavelet is the first known wavelet. Krishnamoorthy et al [23] have shown that the DWT fusion method may minimize the spectral distortion. It also provides better signal to noise ratio than pixel based approach. But the fused images have a less spatial resolution. Naidu et al. [4] have concluded that this method shows good performance if higher level of decomposition is used for image fusion. Dong et al. [6] have concluded that Waveletbased schemes perform better than spatial domain fusion techniques in terms of minimizing color distortion.

\subsection{Kekre's Wavelet Transform Based Fusion Technique}

Kekre et al. [24] have come up with Kekre's Wavelet Transform. Kekre's transform(KT)of size $\mathrm{NxN}$ is used to generate MxM Kekre's Wavelet Transform(KWT) such that $\mathrm{M}=\mathrm{N} * \mathrm{P}$ and $2 \leq \mathrm{P} \leq \mathrm{N}$. Kekre et al.[25] have used kekre's wavelet transform for image fusion. They have shown that KWT can be used for images of any size and fusion results are as good as other algorithms used for image fusion.

\subsection{Kekre's Hybrid Wavelet Transform based Fusion Technique}

Kekre et al. [26] have come up with hybrid wavelet transform. It generates hybrid wavelet transform matrix of size $\mathrm{N} \times \mathrm{N}$ from two orthogonal transform matrices with sizes $\mathrm{P} \times \mathrm{P}$ and $\mathrm{Q} \times \mathrm{Q}$, where $\mathrm{N}=\mathrm{P} \times \mathrm{Q}$. Sarode et al. [27] have come up with a fusion method based on Kekre's Hybrid Wavelet Transform. It decomposes input images using different hybrid transforms like hybrid Kekre-Hadamard hybrid, Kekre-DCT hybrid, DCT-Hadamard hybrid etc. Decomposed images are fused using 'averaging'. Resultant fused transform components are converted to image using inverse transform. Authors have found out that brightness is more in 'kekre-hadamard' wavelet technique. Hybrid 'kekre-DCT' wavelet technique gives best results.

\subsection{Stationary wavelet transform (SWT) Based Image Fusion}

Discrete wavelet transform (DWT) has a drawback"translation-invariance". Stationary avelet transform (SWT)[1] is modified DWT where this drawback is removed by removing the down samplers and up samplers in DWT and up sampling the filter coefficients by a factor of $2(j-$ 1)in the jth level of the algorithm. Borwonwatanadelok et al.[32] , Somkait et al. [33] and kannan et al. [34] have shown that This method provides good result at level 2 of decomposition but it is very time consuming.

\subsection{Stationary Wavelet Transform and Curvelet Transform Based Image Fusion}

Houkui [35] has proposed Stationary Wavelet Transform (SWT) and second generation Curvelet transform based image fusion. In this method, the source images were decomposed using SWT transform and high and low frequency components were respectively acquired, after that the low frequency components were fused using Curvelet transform based image fusion method, high frequency components were fused based on the largest absolute value coefficients, finally the inverse SWT transform were used to get the final fused image. Because of translation invariance, He has shown that this algorithm preserved the advantages of the both, retained image detail and profile information such as edge and effectively avoided block effect based on wavelet transform fusion algorithm and image detail performance deficiencies based on the Curvelet transformation method. The performance index and visual effects are all better than a single SWT algorithm and a separate Curvelet algorithm. 
Table 1: Comparison of Image Fusion Techniques

\begin{tabular}{|c|c|c|}
\hline \multirow{2}{*}{$\frac{\text { Fusion Method }}{\text { Spatial Domain Techniques }}$} & Advantages & Disadvantages \\
\hline & & \\
\hline Averaging Technique $[4,5]$ & \multirow{5}{*}{$\begin{array}{l}\text { All these methods are very simple, easy to understand } \\
\text { and implement. }\end{array}$} & $\begin{array}{l}\text { It reduces the resultant image quality } \\
\text { consequently by introducing noise into } \\
\text { fused image. It leads to unwanted side } \\
\text { effects like reduced contrast. }\end{array}$ \\
\hline $\begin{array}{l}\text { Maximum Pixel Value } \\
\text { Technique }[4,5]\end{array}$ & & \multirow{4}{*}{$\begin{array}{l}\text { These methods produce blurred output } \\
\text { which in turn affects the contrast of the } \\
\text { image. Therefore these techniques are not } \\
\text { suitable for real time applications }\end{array}$} \\
\hline $\begin{array}{l}\text { Minimum Pixel Value } \\
\text { Technique[4,5] }\end{array}$ & & \\
\hline Max- Min Technique[6] & & \\
\hline $\begin{array}{l}\text { Simple Block Replace } \\
\text { Algorithm [6] }\end{array}$ & & \\
\hline $\begin{array}{l}\text { Weighted averaging } \\
\text { technique [7] }\end{array}$ & This method improves the detection reliability. & $\begin{array}{l}\text { It can increase the signal to noise } \\
\text { ratio(SNR) of the fused image. }\end{array}$ \\
\hline $\begin{array}{l}\text { Principal Component } \\
\text { Analysis Algorithm[8-11] }\end{array}$ & $\begin{array}{l}\text { very simple, computationally efficient, faster processing } \\
\text { time and high spatial quality. }\end{array}$ & $\begin{array}{l}\text { Results in spectral degradation and colour } \\
\text { distortion }\end{array}$ \\
\hline IHS[4] & $\begin{array}{l}\text { very simple, computationally efficient and faster } \\
\text { processing and high sharpening ability }\end{array}$ & $\begin{array}{l}\text { It only processes three multispectral } \\
\text { bands and results in colour distortion }\end{array}$ \\
\hline Brovey[5] & $\begin{array}{l}\text { very simple, computationally efficient and faster } \\
\text { processing time. It produces RGB images with higher } \\
\text { degree of contrast }\end{array}$ & Results in colour distortion \\
\hline Guided Filtering[2] & $\begin{array}{l}\text { very simple, computationally efficient, suitable for real } \\
\text { applications }\end{array}$ & \\
\hline \multicolumn{3}{|l|}{ Pyramid Based Fusion } \\
\hline $\begin{array}{l}\text { Fusion using Laplacian/ } \\
\text { Gausian Pyramid [14][15] }\end{array}$ & \multirow{5}{*}{$\begin{array}{l}\text { Pyramid Methods provide good visual quality of an } \\
\text { image for multi-focus images. }\end{array}$} & \multirow{5}{*}{$\begin{array}{l}\text { All pyramid Decomposition Based } \\
\text { Fusion methods produce more or less } \\
\text { similar output. The number of } \\
\text { decomposition levels affects image fusion } \\
\text { result. }\end{array}$} \\
\hline $\begin{array}{l}\text { Fusion using Gradient } \\
\text { Pyramid [16] }\end{array}$ & & \\
\hline $\begin{array}{l}\text { Fusion using Ratio of Low } \\
\text { Pass Pyramid [17] }\end{array}$ & & \\
\hline $\begin{array}{l}\text { Fusion using Filter Subtract } \\
\text { Decimate(FSD) Pyramid [18] }\end{array}$ & & \\
\hline $\begin{array}{l}\text { Fusion using Morphological } \\
\text { Pyramid[19] }\end{array}$ & & \\
\hline \multicolumn{3}{|c|}{ Discrete Transform Based Fusion } \\
\hline $\begin{array}{l}\text { Discrete Cosine Transform } \\
\text { (DCT) Method[21] }\end{array}$ & $\begin{array}{l}\text { It reduces the complexity and decomposed images into } \\
\text { series of waveform. This algorithm can be used for real } \\
\text { applications, }\end{array}$ & $\begin{array}{l}\text { Fused image is not of good quality if } \\
\text { block size is less than } 8 \times 8 \text { or equivalent } \\
\text { to the image size itself. }\end{array}$ \\
\hline $\begin{array}{l}\text { Discrete Wavelet Transform } \\
\text { Method(DWT) with Haar } \\
\text { based fusion } \\
{[22-27]}\end{array}$ & $\begin{array}{l}\text { It provides good quality fused image and better Signal to } \\
\text { Noise Ratio. It also minimizes spectral distortion. }\end{array}$ & $\begin{array}{l}\text { The fused image has less spatial } \\
\text { resolution. }\end{array}$ \\
\hline $\begin{array}{l}\text { Kekre's Wavelet Transform } \\
\text { (KWT) Based Fusion } \\
\text { Technique [28][29] }\end{array}$ & $\begin{array}{l}\text { We can generate various variations of KW transform just } \\
\text { by changing the size of basic Kekre's transform from } \\
\text { which the KW transforms is generated. }\end{array}$ & \\
\hline $\begin{array}{l}\text { Kekre's Hybrid Wavelet } \\
\text { Transform based Fusion } \\
\text { Technique [30][31] }\end{array}$ & $\begin{array}{l}\text { Like KWT it can also be used for images which are not } \\
\text { necessarily integer power of } 2 \text {. }\end{array}$ & \\
\hline $\begin{array}{l}\text { Stationary Wavelet } \\
\text { Transform(SWT) [32-34] }\end{array}$ & $\begin{array}{l}\text { This method provides good result at level } 2 \text { of } \\
\text { decomposition }\end{array}$ & It is time consuming \\
\hline $\begin{array}{l}\text { Stationary Wavelet } \\
\text { Transform and Curvelet } \\
\text { Transform Based Image } \\
\text { Fusion }\end{array}$ & $\begin{array}{l}\text { It has advantages of both SWT and Curvelet } \\
\text { Transform. This algorithm is very well suitable for real } \\
\text { applications. }\end{array}$ & It is time consuming \\
\hline
\end{tabular}




\section{PERFORMANCE MEASURES}

Image fusion should generate an image which is more informative than any of the input images by preserving important information from the source images. It should not add any "new thing" that could mislead the use of fused image. Naidu et al.[4] have concluded that the performance of the image fusion algorithms could be estimated precisely when the ground truth (reference) image is available. The metrics SD, CE and SF would be appropriate metrics to access the quality of the fused image when there is no reference image is available and SF would be the best fusion performance check indicator irrespective of the origin of source images.

Table 2: Performance Measures

\begin{tabular}{|l|l|}
\hline \multicolumn{2}{|l|}{ With reference image } \\
\hline $\begin{array}{l}\text { Root mean square error } \\
\text { (RMSE): }\end{array}$ & $\begin{array}{l}\text { It is computed as the root mean square error of the corresponding pixels in the reference image Ir and the } \\
\text { fused image If. }\end{array}$ \\
\hline $\begin{array}{l}\text { Mean absolute error } \\
\text { (MAE): }\end{array}$ & It is mean absolute error of the corresponding pixels in reference and fused images. \\
\hline $\begin{array}{l}\text { Signal to noise ratio } \\
\text { (SNR) }\end{array}$ & This metric will be high when the reference and fused images are alike. Higher value implies better fusion. \\
\hline $\begin{array}{l}\text { Correlation (CORR): } \\
\text { information }\end{array}$ & $\begin{array}{l}\text { This shows the correlation between the reference and fused image. The ideal value is one when the } \\
\text { reference and fused are exactly alike and it will be less than one when the dissimilarity increases. }\end{array}$ \\
\hline $\begin{array}{l}\text { Mutual } \\
\text { (MI): }\end{array}$ & $\begin{array}{l}\text { It measures the contrast in the fused image. An image with high contrast would have a high standard } \\
\text { deviation. }\end{array}$ \\
\hline Without reference image image quality \\
\hline Standard deviation $(\sigma)$ & $\begin{array}{l}\text { Entropy is used to measure the information content of an image. Entropy is sensitive to noise and other } \\
\text { unwanted rapid fluctuations. An image with high information content would have high entropy }\end{array}$ \\
\hline $\begin{array}{l}\text { Entropy (EN): } \\
\text { Cross entropy(CE): }\end{array}$ & $\begin{array}{l}\text { Cross-entropy evaluates the similarity in information content between input images and fused image. } \\
\text { Fused and reference images containing the same information would have a low cross entropy. }\end{array}$ \\
\hline $\begin{array}{l}\text { Fusion mutual } \\
\text { information (FMI): }\end{array}$ & \begin{tabular}{l} 
It measures the degree of dependence of the two images. A larger measure implies better quality. \\
\hline Special Frequency(SF):
\end{tabular} \\
\hline
\end{tabular}

\section{CONCLUSION}

Image Fusion reads several images of the same scene or objects and retrieves important information from them to put it into a single output image. The resultant fused image is more informative and suitable for visual perception or computer processing than any of the input images which thereby improves quality and applicability of data. It is used in various fields like medical imaging, computer vision, remote sensing, manufacturing process etc. This paper discusses different various spatial and frequency domain techniques and their performance evaluation parameters. Since simple fusion techniques like averaging, minimum, maximum, max-min and simple block replace techniques produce noisy, blurred and low contrast image, they cannot be used for real time applications. Principal component analysis(PCA), IHS and Brovey are very simple, computationally efficient and fast algorithms but these algorithms result in colour distortion. Images fused by PCA have high spatial quality but it results in spectral degradation. IHS also has high sharpening ability. Guided filtering is very simple computational efficient and suitable for real time applications. All pyramid Decomposition Based Fusion methods produce more or less similar output. The number of decomposition levels affects image fusion result. These methods are typically used for multi-focus images. Discrete Cosine Transform (DCT) Method is suitable for real time applications but cannot used with block size less than $8 \times 8$ Kekre's Wavelet Transform Based Fusion and Kekre's Hybrid Wavelet Transform based Fusion Techniques provide better results than other algorithms. . DWT with haar based fusion provides better Signal to Noise Ratio. It also minimizes spectral distortion. However, due to the isotropic nature of wavelet transform, it can't effectively capture the long edge and the curve of the image. Curvelet can solve the blurring of edges and details in wavelet image fusion, but it has no good performance of the local variation of the image. Wavelet transform and Curvelet don't have translation invariance Because of translation invariance; SWT transform has a better description of the details components of images and can effectively preserve the details of the original image compared to the ordinary wavelet transform. Combination of SWT and Curvelet fusion algorithm preserved the advantages of the both, retained image detail and profile information such as edge and effectively avoided block effect based on wavelet transform the fusion algorithm and image detail performance deficiencies based on the Curvelet transformation method. Every algorithm has some advantages and drawbacks. Thus, we can conclude that no fusion algorithm outperforms the others. A combination of qualitative and quantitative assessment approach may be the correct way to find out which fusion algorithm is most appropriate for an application. 


\section{REFERENCES}

[1] L.A. Klein, "Sensor and Data Fusion Concepts and Applications," SPIE Optical Engineering Press, Tutorial Texts, Vol. 14, pp.132-139, 1993.

[2] Shutao Li, "Image Fusion with Guided Filtering", IEEE Transactions On Image Processing, Vol. 22, No. 7, July 2013

[3] Gillespie, A.R., A.B. Kahle, and R.E. Walker, 1987, "Colour enhancement of highly correlated images-II: Channel ratio and chromaticity transformation techniques", Remote Sensing of Environment 22:343365 .

[4] Harris, J.R., R. Murray, and T. Hirose (1990). "IHS transform for the integration of radar imagery with other remotely sensed data." Photogrammetric Engineering and Remote Sensing, Vol. 56, No. 12, pp. 1631-1641.

[5] M. D. Jasiunas, D. A. Kearney, J. Hopf, "Image Fusion for Uninhabited Airborne Vehicles", Proceedings of IEEE International Conference on Field Programmable Technology, 2002, pp. 348-351.

[6] Dong, J., Zhuang, D., Huang, Y., Fu, J., "Survey of Multispectral Image Fusion Techniques in Remote Sensing Applications”, Intech., 1-22 (2011)

[7] Le Song, Yuchi Lin, Weichang Feng, Meirong Zhao "A Novel Automatic Weighted Image Fusion Algorithm", International Workshop on Intelligent Systems and Applications, ISA ,2009, Page(s): 1 - 4

[8] V.P.S. Naidu and J.R. Raol, "Pixel-level Image Fusion using Wavelets and Principal Component Analysis", Defence Science Journal, Vol. 58, No. 3, May 2008, pp. 338-352 Ó 2008, DESIDOC

[9] Lindsay I Smith, "A Tutorial on Principal Component Analysis",http://www.cs.otago.ac.nz/cosc453/student tut orials/principal_components.pdf

[10] "Principal Component Analysis", http://www.cse.unr.edu/ bebis/MathMethods/PCA/lectur e.pdf

[11] F. Sadjadi, "Comparative Image Fusion Analysis", IEEE Computer Society Conference on Computer Vision and Pattern Recognition, Volume 3, Issue , 20-26 June 2005 Page(s): $8-8$

[12] SanjuKumari, Mahesh Malviya, Srikant Lade, "Image Fusion Techniques Based on Pyramid Decomposition", International Journal of Artificial Intelligence and Mechatronics, Volume 2, Issue 4, ISSN 2320 - 5121

[13] Simrandeep Singh, Narwant Singh Grewal, Harbinder Singh,“" Multi-resolution Representation of Multifocus Image Fusion Using Gaussian and Laplacian Pyramids", International Journal of Advanced Research in Computer Science and Software Engineering, Volume 3, Issue 11, November 2013,ISSN: 2277 128X

[14] P. Burt, E. Adelson, "Laplacian pyramid as a compact image code", IEEE Transactions on Communications, Vol.31, No. 4, 1983

[15] Olkkonen, H., and Pesola, P., "Gaussian Pyramid Wavelet Transform for Multiresolution Analysis of
Images", Graphical Models and Image Processing, vol. 58, pp. 394- 398, 1996.

[16] P. Burt, "A gradient pyramid basis for pattern selective image fusion", the Society for Information Displays (SID) International Symposium Digest of Technical Papers, 23,pp. 467-470 (1992).

[17] Toet, A., "Image fusion by a ratio of low-pass pyramid", Pattern Recognition Letters 9, pp. 245-253, 1996.

[18] H. Anderson, "A filter-subtract-decimate hierarchical pyramid signal analyzing and synthesizing technique", U.S. Patent 718 104, 1987

[19] Ramac, L. C., Uner, M. K., Varshney, P. K., "Morphological filters and wavelet based image fusion for concealed weapon detection", Proceedings of SPIE Vol.3376, 1998.

[20] M. D. Jasiunas, D. A. Kearney, J. Hopf, "Image Fusion for Uninhabited Airborne Vehicles", Proceedings of IEEE International Conference on Field Programmable Technology, 2002, pp. 348-351

[21] VPS Naidu, "Discrete Cosine Transform based Image Fusion Techniques", Journal of Communication, Navigation and Signal Processing (January 2012)Vol. 1 , No. 1, pp. 35-45

[22] Rajiv Singh and Ashish Khare, "Multiscale Medical Image Fusion in Wavelet Domain", Hindawi Publishing Corporation, The Scientific World Journal, Volume 2013, Article ID 521034, 10 pages, http://dx.doi.org/10.1155/2013/521034

[23] S. Mallat, "A Wavelet Tour of Signal Processing", Academic Press, 1999

[24] G. Pajares and J. M. dela Cruz, "A wavelet - based image fusion tutorial", Pattern Recognition Journal, vol.37,no.9, Elsevier, pp. 1855-1872,2004.

[25] C. S. Burrus, R. A. Gopinath, H. Guo, J. E. Odegard, and I. W. Selesnick, "Introduction to Wavelets and Wavelet Transforms: A Priimer", PrenticeHall, Upper Saddle River, NJ, USA,1998.

[26] M. Unserand T. Blu, "Wavelet theory demystified", IEEE Transactions on Signal Processing, vol.51, no.2, pp.470-483, 2003

[27] Shivsubramani Krishnamoorthy , K. P. Soman,"Implementation and Comparative Study of Image Fusion Algorithms", International Journal of Computer Applications (0975 - 8887) Volume 9- No.2, November 2010

[28] Dr. H. B. Kekre, Archana Athawale, Dipali Sadavarti "Algorithm to Generate Kekre's Wavelet Transform from Kekre's Transform", International Journal of Engineering Science and Technology, Vol. 2(5), 2010 pp. $756-767$

[29] H. B. Kekre, Tanuja Sarode, Rachana Dhannawat "Implementation and Comparison of different Transform Techniques using Kekre's Wavelet Transform for Image Fusion", International Journal of Computer Applications, Vol. 44, No. 10, 2012, pp. 41-48. 
[30] H. B. Kekre, Rachana Dhannawat, Tanuja Sarode. "Kekre's Hybrid Wavelet Transform Technique with DCT, Walsh, Hartley and Kekre's Transform for Image Fusion", International Journal of Computer Engineering \& Technology, Vol 4,No .1 ,pp. 195-202.

[31] H. B. Kekre, Rachana Dhannawat, TanujaSarode, "Image Fusion Using Kekre's Hybrid Wavelet Transform", International Conference on Communication, Information \& Computing Technology (ICCICT), Oct. 19-20, 2012,Mumbai, India

[32] Pusit Borwonwatanadelok, Wirat Rattanapitak and Somkait Udomhunsakul, "Multi-Focus Image Fusion based on Stationary Wavelet Transform", 2009 International Conference on Electronic Computer Technology. 978-0-7695-3559-3/09 \$25.00 @ 2009 IEEE
[33] Somkait Udomhunsakul, Pradab Yamsang, Suwut Tumthong and Pusit Borwonwatanadelok, "Multiresolution Edge Fusion using SWT and SFM", Proceedings of the World Congress on Engineering 2011 Vol II WCE 2011, July 6 - 8, 2011, London, U.K.

[34] K. Kannan, S. Arumuga Perumal, K. Arulmozhi , "Performance Comparison of various levels of Fusion of Multi-focused Images using Wavelet Transform”, (C2010 International Journal of Computer Applications (0975 8887) Volume 1 - No. 6

[35] Houkui Zhou, "An Stationary Wavelet Transform and Curvelet Transform Based Infrared and Visible Images Fusion Algorithm", International Journal of Digital Content Technology and Applications(JDCTA)Volume6,Number1,January 2012doi:10.4156/jdcta.vol6.issue1.18 